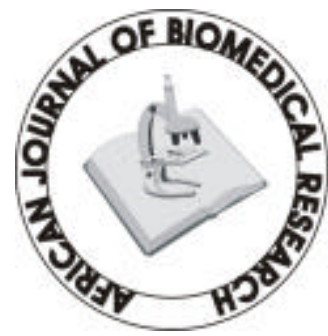

Full-text available at http://www.ajbrui.com \&

http://www.bioline.br/md

Received:

August, 2005

Accepted (Revised):

December, 2006

Published

January, 2006
Full Length Research Article

\section{Starch Based Hydrogel with Potential Biomedical Application as Artificial Skin}

\author{
Kunal Pal ${ }^{\#, ~ \# @ ~ B a n t h i a ~ A . K . ~ a n d ~ * M a j u m d a r ~ D . K . ~}$ \\ \# Materials Science Centre, Indian Institute of Technology, \\ Kharagpur-721302, India. \\ *Delhi Institute of Pharmaceutical Sciences \& Research, \\ Formerly College of Pharmacy, University of Delhi, Pushp Vihar, New Delhi- \\ 110017, India.
}

\section{ABSTRACT}

Hydrogel wound dressing can protect injured skin and keep the wound surface appropriately moist to speed the healing process by absorbing exudates while maintaining the products of tissue repair, including growth factor and lysosomes, in contact with the wound. The design and development of novel membrane of hydrogel prepared by crosslinking of polyvinyl alcohol with starch suspension using glutaraldehyde as a crosslinking agent was attempted. The membrane was characterized by FTIR spectroscopy. The mechanical property of the hydrogel membrane was characterized by tensile tests. The diffusion coefficient of salicylic acid through the membrane was also evaluated using diaphragm cell technique. FTIR spectra of the membrane indicated the absence of free aldehydic groups of glutaraldehyde. The membrane had sufficient strength to be used as artificial skin. At $30^{\circ} \mathrm{C}$, the measured value of the diffusion coefficient of salicylic acid was approximately $4.11 \times 10^{-6} \mathrm{~cm}^{2} / \mathrm{s}$.

(Afr. J. Biomed. Res. 9:23 - 29, 2006)

Keywords: Wound Healing; Hydrogels; Crosslinking; Polyvinyl Alcohol; Starch

**Present/Correspondence address: Prof. A. K. Banthia, Materials Science Centre, Indian Institute of Technology, Kharagpur-721302, INDIA. email:

ajitbanthia2000@yahoo.co.in

Abstracted by:

African Index Medicus (WHO), CAB Abstracts, Global Health Abstracts, Asian Science Index, Index Veterinarius 


\section{INTRODUCTION}

Hydrogels are hydrophilic natured three-dimensional networks, held together by chemical or physical bonds. Water absorbed by hydrogel is not released under ordinary conditions. Hydrophilic groups absorb and store water. If interstitial space exists within the network, water molecules can become trapped and immobilized, filling the available free volume [LaPorte, 1997; Mark et al, 1966]. The water holding property brings about the specific benefit of hydrogels in wound treatment. They immediately function as moist wound dressings and do not need further wound secretions to attain a gelatinous consistency. At the same time they are capable of absorbing the surplus contaminated exudates and safely retaining them within the gel structure. The absorption of secretions causes an expansion of the cross-links in the polymer chains, making room for the inclusion of foreign bodies such as bacteria, detritus, and odor molecules that are irreversibly taken up along with the liquid. The basic physical features of hydrogel dressings can be specifically modified, according to the properties of the polymers used.

Hydrogels save the wound from fluid loss and are capable for providing the lesion with additional moisture, and securely protect it against external noxae [Krasner, 2001; Falanga, 2000; Mulder and Vande Berg, 2002]. Under the dressing a microclimate is developed, that stimulates and regulates all cellular activities and nutritional processes during the individual phases of wound healing. The high moisture content and the soft-elastic, cushioning properties of the hydrogel almost act like a "second skin". The dressing adapts perfectly to the wound and has a light cooling effect, which is agreeable to the patient and helps to ease pain. This feature is of special significance in the treatment of superficial epithelial wounds such as donor sites for split skin grafts, which can often be extremely painful, due to the concomitant exposure of free nerve endings lying underneath the epidermis [Hartmann International, 2005].

The hydrogel dressing removal is almost painless because hydrogel does not adhere to the wound surface. Hydrogel stays permanently moist and can be easily removed after prolonged application without pain and risk of wound irritation [Krasner, 1997; Gruen, 1996; MacLellan, 1994]. Treatment with hydrogels may bring about great relief for both the patient and the nursing staff. Due to the above reasons the hydrogel wound dressings are highly accepted by the patient.

Hydrogels of natural polymers, especially polysaccharides, have been used recently because of their unique advantages. Polysaccharides are, in general, non-toxic, biocompatible, biodegradable, and abundant [Cascone et al, 2001]. However, as polysaccharides dissolve easily in water, cannot form stable hydrogel, an effective method is to make them into a synthesized polymer gel networks to form natural and synthesized polymer blend hydrogels, which is becoming a subject of academic as well as of industrial interest. Hydrogels can be applied as an interface between bone and an implant [Netti et al, 1993], as artificial skin [Young and $\mathrm{Wu}, 1998$ ], as contact lenses [Brinkman et al, 1991], as blood contact materials [Taguchi et al, 1998] and in-controlled release applications for delivery of enzymes, hormones, contraceptives, anticoagulant, etc. [Abusafieh et al, 1997].

Biodegradable polymers such as for instance poly (lactic acid) (PLA), poly (glycolic acid) (PGA) and their respective copolymers are already applied in several drug delivery systems [Zhu et al, 1990; Youxin et al, 1993]. However, only a few attempts [Heller et al, 1990; Pereira et al, 1998] have been reported in trying to use starch-based polymers in these type of applications; despite being well known that they are biodegradable materials [Bastioli, 1995] they have been proposed in several works to be used as biomaterials [Reis et al, 1996; 1997]. Starch is one of the most abundant and cheap polysaccharides. Usually starch includes about $30 \%$ amylose (a linear $\alpha-(1,4)$ glucan) and $70 \%$ amylopectin (dendritically branched version). Chemically modified starches with improved properties are becoming more and more important in industry application not only because they are low in cost, but mainly because the polysaccharide portion of the product is biodegradable. Chemical modification of starch via graft copolymerization of vinyl monomers onto it has been studied widely in recent years [Athawale and Vidyagauri, 1998; Kiatkamjornwong et al, 2000]. But only a few studies on starch polymer blend hydrogels have been reported [Hashim et al, 2000]. In this work PVA/starch blend hydrogels will be prepared by chemical crosslinking technique and efforts will be maid to characterize the hydrogel. 


\section{MATERIALS AND METHODS}

\section{Materials}

Corn starch (CS), salicylic acid (SA), ethanol and glutaraldehyde (GA) were obtained from Loba-Chemie Indoaustranal Co., Mumbai, India. Polyvinyl alcohol (PVA), mol. wt. 125000, was obtained from s.d. finechem. limited, Mumbai, India. Hydrochloric acid 35\% pure was obtained from Merck Limited, Mumbai, India. Double distilled water was used throughout the study.

GA reagent was prepared by mixing $0.5 \mathrm{ml}$ of GA in a solution mixture of $10 \mathrm{ml}$ ethanol and $0.05 \mathrm{ml}$ Hydrochloric acid.

\section{Preparation of hydrogel}

Fifty $\mathrm{ml}$ of $10 \%$ PVA solution was taken in a beaker. To the PVA solution $50 \mathrm{ml}$ of $5 \%$ starch suspension in water was added with constant stirring to get a homogeneous mixture. To this mixture GA reagent $(10.55 \mathrm{ml})$ was added with constant stirring. Care was taken to eliminate entrapment of air bubbles during mixing and the mixture was used to obtain a membrane by the conventional solution casting method at room temperature. The membrane so obtained was named as RT. RT was washed thoroughly with distilled water to wash off the hydrochloric acid and $\mathrm{GA}$, if any. Then the membrane was dried at room temperature.

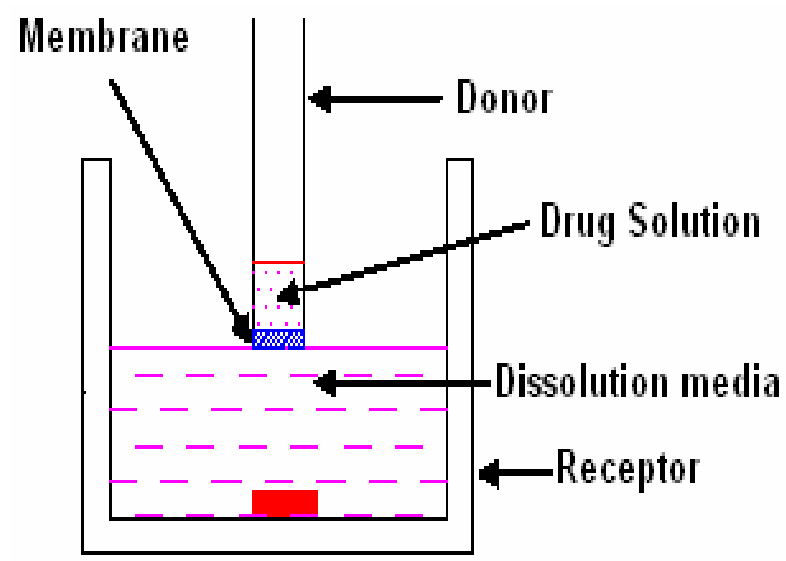

Fig. 1

Diaphragm cell used to measure diffusion coefficient

\section{Characterization}

Corn starch (CS) and PVA were subjected to FTIR spectroscopy in the range of $4000-400 \mathrm{~cm}^{-1}$ as $\mathrm{KBr}$ pellets and the membrane was subjected to Attenuated Total Reflectance FTIR (ATR) spectroscopy in the range of $4000-400 \mathrm{~cm}^{-1}$. FTIR spectrophotometer (NEXUS -870, Thermo Nicolet Corporation) was used for the study.

The raw materials and the patch were subjected to X-ray diffraction (XRD-PW 1700, Philips, US A) using $\mathrm{CuK} \alpha$ radiation generated at $40 \mathrm{KV}$ and $40 \mathrm{~mA}$; the range of diffraction angle was $10.00-70.00^{\circ} 2 \theta$.

The tensile strength of the membrane was determined in the Hounsfield H10KS tensile testing machine. The following test conditions were maintained:

a) Cross-Head Speed: $50 \mathrm{~mm} / \mathrm{min}$

b) Temperature: $18^{\circ} \mathrm{C}$ and

c) Relative Humidity: $60 \%$.

\section{Measurement of diffusion coefficient}

A diaphragm cell shown in Figure 1 was used to measure the diffusion coefficient. The cell consisted of two chambers separated by the hydrogel ( $\sim 0.2-\mathrm{mm}$ thick). The first chamber, donor, contained $5 \mathrm{ml}$ of SA solution in water $(1.6 \mathrm{mg} / \mathrm{ml})$. The other chamber consisted of distilled water (receptor). The system was placed in a constant-temperature water bath. A pipette was used to draw $0.1 \mathrm{ml}$ sample from donor and $1.0 \mathrm{ml}$ sample from the receptor compartment periodically. The samples withdrawn were replaced by same amount of distilled water. The samples were analyzed by acidic ferric chloride solution to determine the concentration of SA in each chamber as a function of time. The diffusion coefficient, $D$, of the drug through the hydrogel was calculated from these results. The experimentation was conducted at $30^{\circ} \mathrm{C}$.

\section{RESULTS AND DISCUSSIONS}

Distinct peaks of hydroxyl groups can be observed at around $3300 \mathrm{~cm}^{-1}$ from the FTIR spectra of CS and PVA (Figure $2 a$ and $2 b$ ). FTIR spectra of RT (Figure $2 c$ ) indicated the presence of free hydroxyl group in the hydrogel membrane. Also there were no free carbonyl group peaks at $1740-1720 \mathrm{~cm}^{-1}$ in the membrane which indicated that all the carbonyl groups of GA have been used up for crosslinking.

\section{XRD Characterization}

(a) $C S$ 

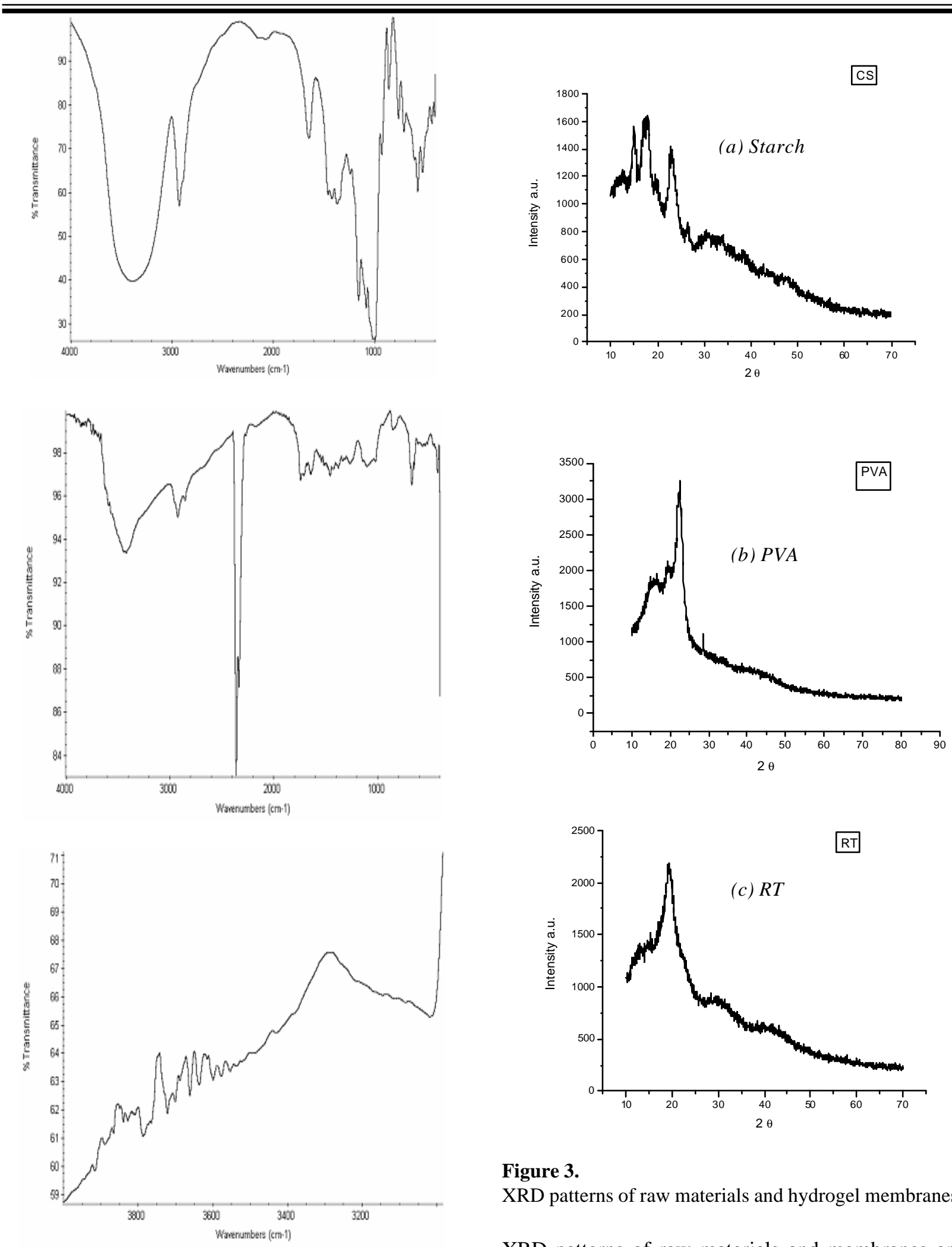

Figure 2.

FTIR spectra of raw materials and hydrogel membranes

Figure 3.

XRD patterns of raw materials and hydrogel membranes

XRD patterns of raw materials and membranes are shown in Figure 3. It can be observed that CS (Figure $3 a$ ) had peaks at $15^{\circ}, 17^{\circ}, 18^{\circ}, 23^{\circ}$ and $26.5^{\circ} 2 \theta$ where the peak at $18^{\circ} 2 \theta$ was most intense while PVA 
(Figure $3 b$ ) had peaks at $19.5^{\circ}, 22.5^{\circ}$, and $28.6^{\circ} 2 \theta$ and the peak at $22.5^{\circ} 2 \theta$ was most intense. In the case of RT (Figure 3c) there was only one peak at $19.25^{\circ}$ $2 \theta$ indicating that the crystallinity of the membrane is mainly due to PVA.

\section{Tensile strength of the membrane}

In order to evaluate the mechanical property of the hydrogel membrane, the tensile strength of the membrane was measured. The tensile strength of the hydrogel membrane was found to be $35.92 \pm 1.87 \mathrm{MPa}$ which is comparable to the failure strength of skin (34 MPa) [Bhat, 2002]. So, the membrane developed could be tried as artificial skin that can give a cushioning effect to the wound.

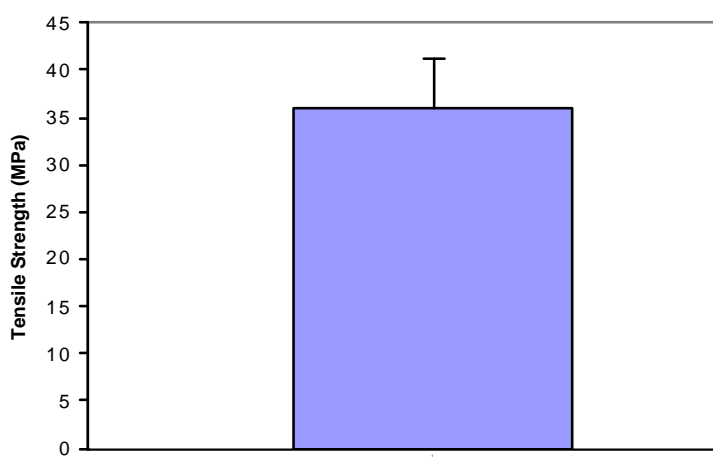

\section{Figure 4.}

Tensile strength of the hydrogel membrane

\section{Diffusion coefficient}

The desired hydrogels can be produced consistently with the technique outlined above. Typical variations of the concentration of SA in the two chambers during a single experiment are shown in Figure 5. As can be expected, the concentration of SA in donor decreases over time, while there is a corresponding increase in the concentration of SA in receptor. At any time, $t$, the concentration values in the two chambers can be used to calculate the diffusion coefficient, $D$, of the drug in the hydrogel from the following equation:

$$
D=\frac{1}{\beta t} x \ln \frac{C_{R}(t)-C_{D}(t)}{C_{R}(0)-C_{D}(0)}
$$

with

$$
\beta=\frac{A_{H}}{W_{H}} x\left[\frac{1}{V_{1}}+\frac{1}{V_{2}}\right]
$$

where: $C_{D}(0)=$ initial concentration of $S A$ in donor; $C_{R}(0)=$ initial concentration of $S A$ in receptor; $C_{D}(t)=$ concentration of $S A$ in donor after time $t$; $C_{R}(t)=$ concentration of $S A$ in receptor after time $t$; $A_{H}=$ effective cross-sectional area of diffusion in the hydrogel sample; $W_{H}=$ width of the hydrogel sample; $V_{R}=$ Volume of $S A$ solution in receptor; and $V_{R}=$ Volume of dissolution media in receptor .

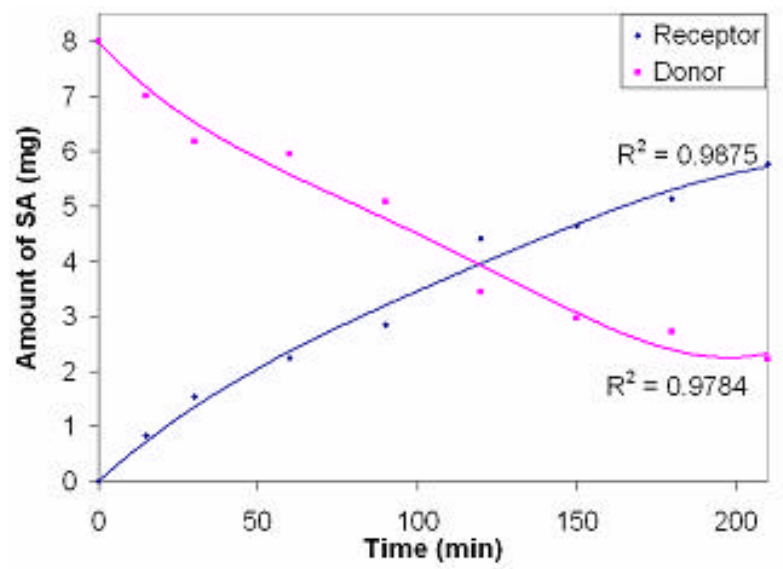

Figure 5.

Diffusion of SA through RT

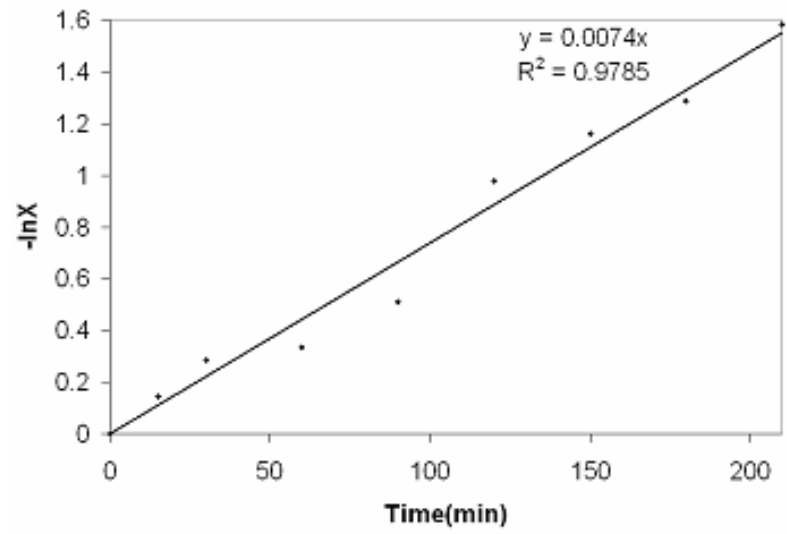

Figure 6.

lnX Vs Time plot, whose slope was used for determining Diffusion coefficient (D)

A plot of $-\ln \frac{C_{D}(t)-C_{R}(t)}{C_{D}(0)-C_{R}(0)}($ denoted by $-\ln \mathrm{X})$ 
with time yielded a straight line as shown in Figure 6. The slope of this line was used to calculate the diffusion coefficient, $D$ as indicated in Eq. 1. The diffusion coefficient, $D$, of the SA through the RT hydrogel membrane was found to be $4.11 \times 10^{-6} \mathrm{~cm}^{2} / \mathrm{s}$. The membrane could be tried as artificial skin and various nutrients/healing factors and medicaments can be delivered directly to the site of action (wound surface) by putting a swab/hydrophilic matrix containing the nutrients/healing factors and medicaments over the artificial skin.

\section{Conclusion}

Membrane obtained by crosslinking of corn starch and PVA with GA showed sufficient strength. FTIR spectra of the membrane indicated the absence of free aldehydic groups of GA thus substantially reducing the chance of toxicity. The XRD studies suggested that the crystallinity imparted in the crosslinked product of CS and PVA was mainly due to PVA. The diffusion coefficient, $D$, of the SA through the cross linked CS and PVA membrane was found to be $4.11 \times 10^{-6} \mathrm{~cm}^{2} / \mathrm{s}$. The prepared hydrogel membrane could be tried as artificial skin and at the same time various nutrients/healing factors and medicaments can be delivered directly to the site of action.

\section{Acknowledgement}

The authors are thankful to Indian Institute of Technology, Kharagpur, India for funding the research. The first author is also grateful to his lab-technician and lab-mates (Mr. N. K. Mallick, Mr. A. H. Bhat, Mr. Arfat Anis, Mr. Dibakar Behara, Ms. H. Satpathee, Mr. R. Dasgupta and Mr. P. Pawar) for their constant encouragement and support during the completion of the work.

\section{REFERENCES}

Abusafieh A., S. Siegler and S.R. Kalidindi (1997): Development of self-anchoring bone implants. I. Proces sing and material characterization. J Biomed Res 38, pp. 314-327.

Athawale V.D. and Vidyagauri L (1998): Graft copolymerization onto starch. II. Grafting of acrylic acid and preparation of it's hydrogels. Carbohydrate Polymers 35, pp. 21-27.

Bastioli C. In: Degradable polymer-principles and applications. London: Chapman and Hall, 1995. p. 112. Bhat S.V. (2002): Biomaterials, pp.106, Narosa Publishing House, New Delhi, India.
Brinkman E., L. van der (1991): Does and A. Bantjes, Poly(vinyl alcohol)-heparin hydrogels as sensor catheter membranes. Biomaterials 12, pp. 6370.

Cascone M.G., Barbani N., Cristallini C., Giusti P., Ciardelli G. and Lazzeri L (2001): Bioartificial polymeric materials based on polysaccharides. Journal of Biomaterials Science, Polymer Edition 12 3, pp. 267-281.

Chen J., Jo S. and Park K (1995): Polysaccharide hydrogels for protein drug delivery. Carbohydrate Polymers 28, pp. 69-76.

Falanga, V (2000): Classifications for wound bed preparation and stimulation of chronic wounds. Wound Repair Regen Sep-Oct;8(5):347-52.

Gruen RL, Chang S, MacLellan DG (1996): Optimizing the hospital management of leg ulcers. Aust N Z J Surg;66:171-4.

Hartman International (2005): Wound management: The stage adapted use of hydro- active wound dressings. http://www.hartmann-online.de

Hashim K., Dahlan K.Z. and Noordin N.M (2000): Hydrogel of sago starch/water-soluble polymers by electron beam irradiation technique. International Symposium on Radiation Technology in Emerging Industrial Applications, pp. 79-80. International Atomic Energy Agency(IAEA)-SM-365.

Heller J., Pangburn S.H. and Roskos K.V (1990): Development of enzymatically degradable protective coatings for use in triggered drug delivery systems: derivatized starch hydrogels. Biomaterials 11, pp. 345-350.

Kiatkamjornwong S., Chomsaksakul W. and Sonsuk M (2000): Radiation modification of water absorption of cassava starch by acrylic acid/acrylamide. Radiation Physics and Chemistry 59, pp. 413-427.

Krasner D (1997): Chronic wound pain. In: Krasner D, Kane D, editors. Chronic wound care: a clinical source book for healthcare professionals. 2nd ed. Wayne (PA): Health Management Publications; pp. 336-43.

Krasner DL.(2001): How to prepare the wound bed. Ostomy Wound Manage (united States), 47(4) pp. 5961.

LaPorte RJ ((1997): In: Hydrophilic polymer coating for medical devices. Lancaster, USA: Technomic Publishing Co, pp. 19-50.

Mack E.J., T. Okano and S.W. Kim. In: N.A. 
Peppas (1988) Editor, Hydrogels in medicine and pharmacy-polymers vol. II, CRC Press, Boca Raton, USA (1988), pp. 65.

MacLellan DG (1994): Chronic leg ulceration - the hidden epidemic. Med J Aust 1994;161:619-21.

Mulder GD, Vande Berg JS (2002): Cellular senescence and matrix metalloproteinase activity in chronic wounds. Relevance to debridement and new technologies. J Am Podiatr Med Assoc Jan;92(1):34-7. Netti P.A., Shelton J.C., Revell P.A., Pirie C., S. Smith, Ambrosio L., Nicolais L. and Bonfield W (1993): Hydrogels as an interface between bone and an implant. Biomaterials 14, pp. 1098-1104.

Pereira C.S., A.M. Cunha, R.L. Reis, B. Vazquezand J. San Roman (1998): New starchbased thermoplastic hydrogels for use as bone cements or drug delivery carriers. J Mater Sci: Mater Med 9, pp. 825-833.

Reis R.L., Cunha A.M. and Bevis M.J (1997): Using non-conventional processing routes to develop anisotropic and biodegradable composites of starch based thermoplastic reinforced with bone-like ceramics. Med Plast Biomater 4, pp. 46-50.

Reis R.L., Cunha A.M., Allan P.S. and Bevis M.J
(1995): Mechanical behaviour of injection moulded starch-based polymers. Polym Adv Technol 7, pp. 784-790.

Taguchi T., Kishida A., Sakamoto N. and Akashi $M$ (1998): Preparation of a novel functional hydrogel consisting on sulphated glycoside-bearing polymer: activation of basic fibroblast growth factor. $J$ Biomed Mater Res 41, pp. 386-391.

Young CD, JR Wu, TL Tsou (1998):. Fabrication and characteristics of polyHEMA artificial skin with improved tensile properties. J Membr Sci;146:83-93.

Youxin L. and KisseT.l (1993): Synthesis and properties of biodegradable ABA triblock copolymers consisting of poly( -lactic acid) or poly( -lacticcoglycolic acid) A-blocks attached to central poly(oxyethylene) Bblocks. J Control Rel 27, pp. 247-257.

Zhu K.J., Xiangzhou L. and Shilin Y (1990): Preparation, characterization, and properties of polylactide (PLA)-poly(ethylene glycol) (PEG) copolymers: a potential drug delivery carrier. J Appl Polym Sci 39, pp. 1-9 